Georgian Mathematical Journal

Volume 13 (2006), Number 1, 101-108

\title{
ON SOME MUTUAL POSITIONS OF HYPERPLANES IN A FINITE-DIMENSIONAL AFFINE SPACE
}

\author{
ALEXANDER KHARAZISHVILI
}

\begin{abstract}
Several combinatorial questions and facts connected with certain types of mutual positions of finitely many hyperplanes in a finite-dimensional affine space are considered. An application of one of such facts to a multidimensional version of the well-known Sylvester theorem is presented.
\end{abstract}

2000 Mathematics Subject Classification: 52A10, 52C10, 52C35.

Key words and phrases: Affine space, hyperplane, admissible set, admissible simplex, Sylvester theorem.

Let $E$ be a finite-dimensional affine space and let $n=\operatorname{dim}(E)$. Without loss of generality, we may identify $E$ with the canonical product space $R^{n}$ (where $R$ denotes, as usual, the real line).

Let $X$ be a subset of $E$. We say that $X$ is admissible if, for any pairwise distinct points $x_{1} \in X, x_{2} \in X, \ldots, x_{n} \in X$, there exists a unique hyperplane in $E$ containing all these points. We denote that hyperplane by $L\left(x_{1}, x_{2}, \ldots, x_{n}\right)$. Also, for any two distinct points $x$ and $y$ in $E$, we denote by $l(x, y)$ the straight line passing through these points.

Let $\mathcal{L}$ be an injective family of hyperplanes in $E$. We say that this family is admissible if, for any pairwise distinct hyperplanes $L_{1} \in \mathcal{L}, L_{2} \in \mathcal{L}, \ldots$, $L_{n} \in \mathcal{L}$, the corresponding exterior normal vectors $e\left(L_{1}\right), e\left(L_{2}\right), \ldots, e\left(L_{n}\right)$ are linearly independent. In that case, we have

$$
L_{1} \cap L_{2} \cap \cdots \cap L_{n}=\{x\}
$$

for some uniquely determined point $x \in E$. We denote $x=x\left(L_{1}, L_{2}, \ldots, L_{n}\right)$ and define the set of points

$$
X(\mathcal{L})=\left\{x\left(L_{1}, L_{2}, \ldots, L_{n}\right):\left\{L_{1}, L_{2}, \ldots, L_{n}\right\} \subset \mathcal{L}\right\} .
$$

If $x \in X(\mathcal{L})$, then we say that $x$ is a point associated with a given family $\mathcal{L}$.

Let $S=\left[x_{1}, x_{2}, \ldots, x_{n+1}\right]$ denote the $n$-dimensional simplex in $E$ whose vertices are $x_{1}, x_{2}, \ldots, x_{n+1}$. We say that $S$ is associated with $\mathcal{L}$ if each facet (i.e., each $(n-1)$-dimensional face) of $S$ is carried by a hyperplane belonging to $\mathcal{L}$. Obviously, if $S$ is associated with $\mathcal{L}$, then all its vertices $x_{1}, x_{2}, \ldots, x_{n+1}$ are also associated with $\mathcal{L}$.

We say that a simplex $S=\left[x_{1}, x_{2}, \ldots, x_{n+1}\right]$ is admissible for $\mathcal{L}$ if the following two conditions hold:

(a) $S$ is associated with $\mathcal{L}$;

(b) there exists a point $x$ also associated with $\mathcal{L}$ and belonging to $] x_{i}, x_{j}[$ where $x_{i}$ and $x_{j}$ are some two distinct vertices of $S$. 
Suppose now that $\mathcal{L}$ is an injective finite family of hyperplanes in $E$ and $\operatorname{card}(\mathcal{L})=k$. Obviously, there are only finitely many purely combinatorial possibilities for mutual positions (arrangements) in $E$ of elements of $\mathcal{L}$. Briefly, there are only finitely many combinatorial types of mutual positions of $k$ hyperplanes in $E$. The total number of these combinatorial types is denoted by $p_{n}(k)$.

The problem of finding an exact formula for $p_{n}(k)$ is very difficult. In this context, let us note that if $n=1$, then $p_{n}(k)=1$ for all natural numbers $k$. If $n=2$, then for small natural numbers $k$ we have

$$
p_{2}(0)=1, p_{2}(1)=1, p_{2}(2)=2, p_{2}(3)=4, p_{2}(4)=9, p_{2}(5)=47, \ldots .
$$

Let us consider more thoroughly the case $n=2, k=4$. In this case, all combinatorial types of mutual positions of four straight lines $l_{1}, l_{2}, l_{3}, l_{4}$ on the affine plane are well known and are presented below (see Fig. 1).
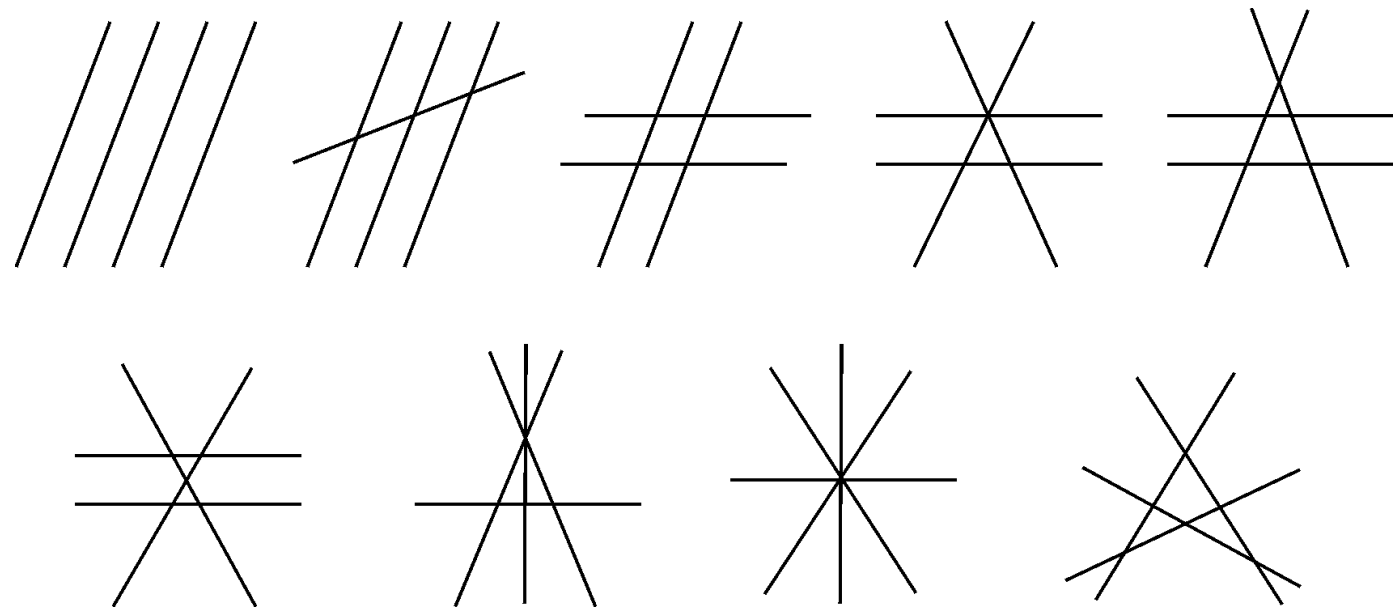

Fig. 1

We readily observe that if any two of the lines $l_{1}, l_{2}, l_{3}, l_{4}$ are not parallel and these four lines have no common point, then there is a triangle admissible for the family $\mathcal{L}=\left\{l_{1}, l_{2}, l_{3}, l_{4}\right\}$. This simple geometrical fact is shown in Figure 2 below.
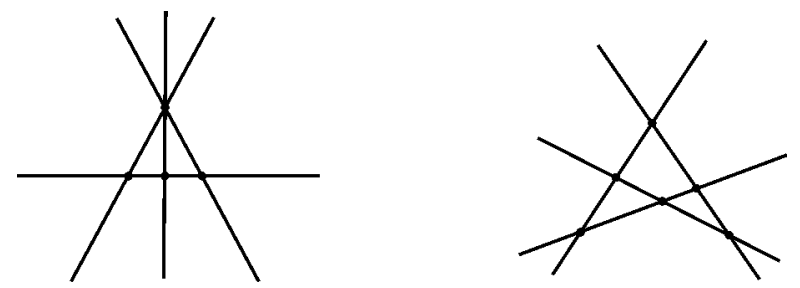

Fig. 2

The above observation is the starting point for our further constructions.

Lemma 1. Let $\operatorname{dim}(E)=n>0$, let $\mathcal{L}=\left\{L_{1}, L_{2}, \ldots, L_{n+2}\right\}$ be an admissible family of hyperplanes in $E$ and suppose that $\cap \mathcal{L}=\varnothing$. Then there exists at least one admissible simplex for $\mathcal{L}$. 
Proof. We use the method of induction on $n$. The case $n=1$ is trivial. Suppose that our assertion is valid for all natural numbers $m<n$ and let us establish its validity for $m=n$. Take, in a space $E$ with $\operatorname{dim}(E)=n$, any admissible family $\mathcal{L}=\left\{L_{1}, L_{2}, \ldots, L_{n+2}\right\}$ of hyperplanes such that $\cap \mathcal{L}=\varnothing$ and define:

$$
\begin{gathered}
P_{1}=L_{1} \cap L_{n+2}, P_{2}=L_{2} \cap L_{n+2}, \ldots, P_{n+1}=L_{n+1} \cap L_{n+2}, \\
\mathcal{L}^{\prime}=\left\{P_{1}, P_{2}, \ldots, P_{n+1}\right\} .
\end{gathered}
$$

Clearly, $\mathcal{L}^{\prime}$ is an admissible family of hyperplanes in the affine space $L_{n+2}$ such that

$$
\operatorname{card}\left(\mathcal{L}^{\prime}\right)=n+1, \cap \mathcal{L}^{\prime}=\varnothing .
$$

Since $\operatorname{dim}\left(L_{n+2}\right)=n-1<n$, we can apply the inductive assumption to $\mathcal{L}^{\prime}$. Consequently, there exists an $(n-1)$-dimensional simplex

$$
S^{\prime}=\left[x_{1}, x_{2}, \ldots, x_{n}\right] \subset L_{n+2}
$$

which is admissible for $\mathcal{L}^{\prime}$. Let $P_{i_{1}}, P_{i_{2}}, \ldots, P_{i_{n}}$ be all those hyperplanes in $L_{n+2}$ which carry the facets of $S^{\prime}$, and let

$$
x=x\left(L_{i_{1}}, L_{i_{2}}, \ldots, L_{i_{n}}\right) .
$$

It is obvious that the point $x$ does not belong to $L_{n+2}$. Therefore we can consider, in $E$, the $n$-dimensional simplex $S=\left[x, x_{1}, x_{2}, \ldots, x_{n}\right]$. An easy verification shows that $S$ is admissible for the original family $\mathcal{L}$. This completes the proof of the lemma.

Remark 1 . Let us denote by $s(\mathcal{L})$ the number of admissible simplices for a given admissible family $\mathcal{L}=\left\{L_{1}, L_{2}, \ldots, L_{n+2}\right\}$ of hyperplanes in $E$, satisfying the relation $\cap \mathcal{L}=\varnothing$. It would be interesting to find some good lower bounds for $s(\mathcal{L})$. The inequality $s(\mathcal{L}) \geq 1$ (stated by Lemma 1 ) is completely sufficient for our further consideration. If $\operatorname{dim}(E)=n=2$, then a situation may occur, where $s(\mathcal{L})=1$ (see Fig. 2). Also, it is not difficult to show that if $\operatorname{dim}(E)=n \geq 3$, then $s(\mathcal{L}) \geq 2$.

Lemma 2. Let $S=\left[x_{1}, x_{2}, \ldots, x_{n+1}\right]$ be the $n$-dimensional simplex in $E$ with vertices $x_{1}, x_{2}, \ldots, x_{n+1}$ and let $x^{\prime}$ be a point belonging to the edge $\left[x_{j}, x_{k}\right]$ of $\left[x_{1}, x_{2}, \ldots, x_{n}\right]$ and distinct from all vertices $x_{1}, x_{2}, \ldots, x_{n}$. Finally, let $\Gamma$ be a hyperplane in E such that:

1) $\Gamma$ passes through $x^{\prime}$;

2) $\Gamma$ does not contain $x_{n+1}$;

3) $\Gamma$ does not contain the edge $\left[x_{j}, x_{k}\right]$.

Then there exist an index $i \in\{1,2, \ldots, n\}$ and a point $z$ such that

$$
z \in \Gamma \cap] x_{i}, x_{n+1}[\text {. }
$$

In particular, we have the inequality

$$
\operatorname{dist}\left(z, L\left(x_{1}, x_{2}, \ldots, x_{n}\right)\right)<\operatorname{dist}\left(x_{n+1}, L\left(x_{1}, x_{2}, \ldots, x_{n}\right)\right) .
$$


The proof of Lemma 2 can easily be carried out by induction on $n$. Omitting details, we only note that, for $n=2$, the formulation of this lemma is very similar to the well-known Pasch axiom from elementary geometry. The above-mentioned axiom plays an essential role in studyings the foundations of geometry (cf. [1]).

Now, let us give an application of Lemmas 1 and 2 to the problem concerning certain mutual positions of hyperplanes (or points) in a finite-dimensional affine space $E$. Problems and questions of this kind are typical in combinatorial and discrete geometry (see, for instance, [2], [3], [4]).

Theorem 1. Let $\operatorname{dim}(E)=n>1$ and let $\mathcal{L}$ be a finite admissible family of affine hyperplanes in $E$, satisfying the following conditions:

1) $\operatorname{card}(\mathcal{L}) \geq n$;

2) for any pairwise distinct hyperplanes $L_{1} \in \mathcal{L}, L_{2} \in \mathcal{L}, \ldots, L_{n} \in \mathcal{L}$, there exists at least one hyperplane $L \in \mathcal{L}$ such that

$$
L \neq L_{1}, L \neq L_{2}, \ldots, L \neq L_{n}, L \cap L_{1} \cap L_{2} \cap \cdots \cap L_{n} \neq \varnothing .
$$

Then we have $\cap \mathcal{L} \neq \varnothing$.

Proof. Suppose otherwise, i.e., suppose that $\cap \mathcal{L}=\varnothing$. Then there are a point $x$ associated with $\mathcal{L}$ and a hyperplane $L \in \mathcal{L}$ for which $\operatorname{dist}(x, L)>0$. We may assume without loss of generality that $\operatorname{dist}(x, L)$ takes a minimal possible value. According to the definition of $x$, there are some pairwise distinct hyperplanes $L_{1}, L_{2}, \ldots, L_{n}$ from $\mathcal{L}$ such that

$$
\{x\}=L_{1} \cap L_{2} \cap \cdots \cap L_{n} .
$$

Further, by the assumption of the theorem, there exists a hyperplane $L_{n+1} \in \mathcal{L}$ passing through $x$ and distinct from all hyperplanes $L_{1}, L_{2}, \ldots, L_{n}$. Let us put

$$
P_{1}=L_{1} \cap L, P_{2}=L_{2} \cap L, \ldots, P_{n+1}=L_{n+1} \cap L .
$$

The family $\mathcal{P}=\left\{P_{1}, P_{2}, \ldots, P_{n+1}\right\}$ of hyperplanes in the $(n-1)$-dimensional affine space $L$ is admissible and $\cap \mathcal{P}=\varnothing$. Applying Lemma 1 to $\mathcal{P}$, we obtain an $(n-1)$-dimensional simplex $S^{\prime}=\left[x_{1}, x_{2}, \ldots, x_{n}\right] \subset L$ admissible for $\mathcal{P}$. Denote by $x^{\prime}$ a point associated with $\mathcal{P}$, belonging to some edge $\left[x_{j}, x_{k}\right]$ of $S^{\prime}$ and distinct from all vertices of $S^{\prime}$. We may write

$$
\left\{x^{\prime}\right\}=L_{i_{1}} \cap L_{i_{2}} \cap \cdots \cap L_{i_{n-1}} \cap L,
$$

where $i_{1}, i_{2}, \ldots, i_{n-1}$ are some pairwise distinct indices from the segment $[1, n+1]$ of natural numbers. Again, by the assumption of the theorem, there exists a hyperplane $\Gamma \in \mathcal{L}$ passing through $x^{\prime}$ and satisfying the relations

$$
\Gamma \neq L_{i_{1}}, \Gamma \neq L_{i_{2}}, \ldots, \Gamma \neq L_{i_{n-1}}, \Gamma \neq L
$$

Observe that $\Gamma$ does not contain the edge $\left[x_{j}, x_{k}\right]$ and does not pass through $x$ (since our family $\mathcal{L}$ is admissible). So we may apply Lemma 2 to $\Gamma$ and to the $n$-dimensional simplex $S=\left[x_{1}, x_{2}, \ldots, x_{n}, x\right]$. In this way we obtain that

$$
\Gamma \cap] x, x_{i}[\neq \varnothing
$$


for some index $i \in\{1,2, \ldots, n\}$. If $z \in \Gamma \cap] x, x_{i}$, then we obviously have

$$
0<\operatorname{dist}(z, L)<\operatorname{dist}(x, L) \text {. }
$$

On the other hand, it can be easily verified that $z$ is a point associated with $\mathcal{L}$. So we come to a contradiction with the choice of $x$, which completes the proof of Theorem 1.

Remark 2. It is not difficult to give a direct proof of Theorem 1 by applying the method of induction on $n=\operatorname{dim}(E)$ and using the fact that if a family $\left\{\Gamma_{1}, \Gamma_{2}, \ldots, \Gamma_{k}\right\}$ of hyperplanes in the space $R^{n}$ is admissible, then the family

$$
\left\{\Gamma_{1} \cap \Gamma_{k}, \Gamma_{2} \cap \Gamma_{k}, \ldots, \Gamma_{k-1} \cap \Gamma_{k}\right\}
$$

of hyperplanes in the $(n-1)$-dimensional affine space $\Gamma_{k}$ is admissible, too.

The case $n=2$ is considered, e.g., in [2]. More precisely, suppose that $\mathcal{L}$ is a finite family of straight lines in $R^{2}$ satisfying the condition: for any two distinct lines $l_{1} \in \mathcal{L}$ and $l_{2} \in \mathcal{L}$, the relation $l_{1} \cap l_{2} \neq \varnothing$ implies the existence of a third line $l_{3} \in \mathcal{L}$ such that $l_{1} \cap l_{2} \cap l_{3} \neq \varnothing$. Then either $\cap \mathcal{L} \neq \varnothing$ or all lines from $\mathcal{L}$ are parallel to each other (cf. [2]).

For our further purposes, we need to recall the notion of a polarity correspondence between points and hyperplanes in the Euclidean space $R^{n}$.

Let $y \in R^{n}$ be a fixed point and let $k$ be a strictly positive real number. For each point $x \in R^{n}$ distinct from $y$, consider the point $x^{\prime} \in l(x, y)$ such that

$$
y \notin\left[x, x^{\prime}\right],\|y-x\| \cdot\left\|y-x^{\prime}\right\|=k .
$$

Let $\Gamma(x)$ denote the hyperplane in $R^{n}$ passing through $x^{\prime}$ and orthogonal to $l(x, y)$. We thus obtain the bijective mapping

$$
\phi_{y}: x \rightarrow \Gamma(x)
$$

between the set $R^{n} \backslash\{y\}$ and the family of all those hyperplanes in $R^{n}$ which do not pass through $y$. The geometric properties of $\phi_{y}$ are well known. In particular, points $x_{1}, x_{2}, \ldots, x_{k}$ distinct from $y$ lie in an affine hyperplane $L$ (not containing $y$ ) if and only if the corresponding affine hyperplanes $\phi_{y}\left(x_{1}\right), \phi_{y}\left(x_{2}\right), \ldots$, $\phi_{y}\left(x_{k}\right)$ have a common point (which, in fact, coincides with $\left.\phi_{y}^{-1}(L)\right)$.

The polarity between points and hyperplanes in $E$ enables us to transform an admissible subset of $E$ with small cardinality into an admissible family of hyperplanes in the same $E$. More precisely, we have

Lemma 3. Let $\operatorname{dim}(E)=n \geq 1$ and let $X$ be an admissible subset of $E$ with $\operatorname{card}(X)<\mathbf{c}$, where $\mathbf{c}$ denotes the cardinality of the continuum. Then there exists a point $y \in E$ such that the family of hyperplanes $\left\{\phi_{y}(x): x \in X\right\}$ is admissible, too.

Proof. For any pairwise distinct points $x_{1}, x_{2}, \ldots, x_{n}$ from $X$, denote by $L\left(x_{1}, x_{2}, \ldots, x_{n}\right)$ the unique affine hyperplane determined by these points. Also, put

$$
\mathcal{L}_{X}=\left\{L\left(x_{1}, x_{2}, \ldots, x_{n}\right):\left\{x_{1}, x_{2}, \ldots, x_{n}\right\} \subset X\right\}
$$


Then, according to our assumption, $\operatorname{card}\left(\mathcal{L}_{X}\right)<\mathbf{c}$. This implies that the family $\mathcal{L}_{X}$ does not cover the space $E$, so there exists a point $y \in E \backslash \cup \mathcal{L}_{X}$. It can be easily seen that $y$ is the desired point.

Lemma 3 and Theorem 1 enable us to prove some multi-dimensional analogue of the Sylvester theorem on collinear points. This theorem was first formulated in [5]. For further extensions and generalizations, see, e.g., [6],[7] and [8].

Theorem 2. Let $\operatorname{dim}(E)=n>1$ and let $X$ be a finite admissible subset of $E$ satisfying the condition: for any pairwise distinct points $x_{1}, x_{2}, \ldots, x_{n}$ from $X$, we have

$$
\operatorname{card}\left(X \cap L\left(x_{1}, x_{2}, \ldots, x_{n}\right)\right) \geq n+1 .
$$

Then there exists a hyperplane in $E$ containing $X$.

Proof. We may assume without loss of generality that $\operatorname{card}(X) \geq n$. According to Lemma 3, there exists a point $y \in E$ such that the family of hyperplanes $\left\{\phi_{y}(x): x \in X\right\}$ is admissible. This family also satisfies the assumptions of Theorem 1. Consequently, $\cap\left\{\phi_{y}(x): x \in X\right\} \neq \varnothing$. Take a point $z$ from $\cap\left\{\phi_{y}(x): x \in X\right\}$ and define $L=\phi_{y}(z)$. Then we readily claim that $X \subset L$, which ends the proof of Theorem 2.

Remark 3. Some other versions of Theorem 1 (Theorem 2) can be formulated and proved without difficulties. For example, let $\mathcal{L}$ be a finite family of hyperplanes in $E$ such that, for any two distinct and nonparallel hyperplanes $L_{1}$ and $L_{2}$ from $\mathcal{L}$, there exists a third hyperplane $L_{3} \in \mathcal{L}$ satisfying

$$
L_{1} \neq L_{3}, L_{2} \neq L_{3}, L_{1} \cap L_{2} \subset L_{3} .
$$

Then either all members of $\mathcal{L}$ are parallel to each other or there exists an $(n-2)$ dimensional affine linear manifold $M \subset E$ such that $M \subset \cap \mathcal{L}$. The proof of this fact can be carried out by induction on $\operatorname{dim}(E)$.

It would be interesting to find the most general form of the Sylvester theorem for the space $R^{n}$ (formulated in terms of $k$-dimensional affine linear submanifolds of $R^{n}$, where $k$ ranges over the set $\left.\{0,1, \ldots, n-1\}\right)$.

Theorem 2 implies the following statement.

Theorem 3. Let $X$ be a finite set of points of $R^{n}(n \geq 2)$ in general position such that, for any $(n-1)$-dimensional sphere $T$ in $R^{n}$, the relation $\operatorname{card}(T \cap X) \geq n+1$ implies the relation $\operatorname{card}(T \cap X) \geq n+2$. Then there exists an $(n-1)$-dimensional sphere in $R^{n}$ containing $X$.

Proof. Actually, Theorem 3 can easily be obtained from Theorem 2 by using the standard technique, namely, the inversion of $R^{n}$ whose pole coincides with one of the elements of $X$ and whose coefficient is an arbitrary strictly positive number (cf. [2]).

Remark 4. We say that a set $T \subset R^{2}$ is a generalized circumference in $R^{2}$ if $T$ is either a circumference or a straight line. In other words, we treat each straight line in $R^{2}$ as a circumference of infinite radius. For $R^{2}$, we have the following 
analog of Theorem 3 in terms of generalized circumferences: if $X$ is a finite subset of $R^{2}$ such that the relation $\operatorname{card}(X \cap T) \geq 3 \operatorname{implies} \operatorname{card}(X \cap T) \geq$ 4 for every generalized circumference $T \subset R^{2}$, then $X$ is contained in some generalized circumference.

Theorem 4. Let $\mathcal{L}$ be a family of straight lines in $R^{2}$ satisfying the following condition: if any three pairwise distinct lines $l_{1}, l_{2}, l_{3}$ from $\mathcal{L}$ are tangent to some circumference $T \subset R^{2}$, then there exists one more line $l_{4} \in \mathcal{L}$ which is also tangent to the same $T$.

Then at least one of the next three relations holds:

1) all lines from $\mathcal{L}$ are parallel to each other;

2) all lines from $\mathcal{L}$ have a common point;

3) the family $\mathcal{L}$ is infinite.

Proof. Considering all possible combinatorial types of mutual positions of three straight lines in the plane, we observe that if some three pairwise distinct lines are given in $R^{2}$ and are tangent to a circumference $T$ lying in $R^{2}$, then the radius length of $T$ can take at most four values. Starting with this observation, suppose to the contrary that none of the above relations 1) - 3) is valid and consider a circumference $T_{0} \subset R^{2}$ of the smallest radius such that there are three pairwise distinct lines from $\mathcal{L}$ tangent to $T_{0}$. A simple argument shows that the existence of $T_{0}$ leads to a contradiction, which yields the desired result.

\section{REFERENCES}

1. D. Hilbert, Foundations of geometry. (Russian) Translation from the German with comments by P. K. Rashevskii. Moscow-Leningrad, 1948.

2. H. Hadwiger and H. Debrunner, Combinatorial geometry in the plane. Translated by Victor Klee. With a new chapter and other additional material supplied by the translator. Holt, Rinehart and Winston, New York, 1964.

3. V. G. Boltjanskil̆, Theorems and problems in combinatorial geometry. (Russian) Nauka, Moscow, 1965.

4. A. B. Kharazishvili, Introduction to combinatorial geometry. (Russian) Tbilis. Gos. Univ., Tbilisi, 1985.

5. J. J. Sylvester, Question 11851. Educational Times LIX(1893), 98.

6. N. G. DE BruiJn and P. Erdös, On a combinatorial problem. Nederl. Akad. Wetensch., Proc. 51 (1948), 1277-1279 = Indagationes Math. 10 (1948), 421-423.

7. H. S. M. Coxeter, A problem of collinear points. Amer. Math. Monthly 55(1948), $26-28$.

8. F. Herzog and L. M. Kelly, A generalization of the theorem of Sylvester. Proc. Amer. Math. Soc. 11(1960), 327-331.

(Received 20.06.2005) 
Author's address:

A. Razmadze Mathematical Institute

Georgian Academy of Sciences

1, Aleksidze St., Tbilisi 0193

Georgia

I. Vekua Institute of Applied Mathematics

Tbilisi State University

2, University St. Tbilisi 0143

Georgia

E-mail: kharaz2@yahoo.com 